\title{
Nächtliche Bettnässer: Am nachhaltigsten wirkt die Klingelmatte
}

Eine kanadische Autorin hat die Gründe für die Enuresis nocturna sowie die aktuellen Behandlungsmöglichkeiten zusammengefasst. Wichtig ist vor allem, dass sich das oft in seinem Selbstwertgefühl angeschlagene Kind von seinen Eltern verstanden und unterstützt fühlt. Gleichzeitig sollte die Zahl der Untersuchungen auf ein Minimum beschränkt werden.

D ie Enuresis nocturna ist definiert als nächtliches Bettnässen von fünfjährigen und älteren Kindern. Einer amerikanischen Studie zufolge sind von dem Problem überwiegend Jungs betroffen. Mit 6,2\% bei den Acht- bis Elfjährigen liegen sie weit vor dem Anteil der Mädchen mit 2,5\%. Insgesamt scheinen drei Faktoren die Neigung zum nächtlichen Einnässen zu begünstigen: eine übermäßige nächtliche Urinbildung, eine überaktive Blase und dass der Harndrang im Schlaf nicht bemerkt wird. Viele Studien haben diese Mechanismen bestätigt, dennoch existiert bislang keine schlüssige Theorie zur Pathophysiologie des Bettnässens.

Am Anfang stehen eine gründliche Anamnese sowie die gezielte körperliche Untersuchung. Gefragt werden sollte nach Symptomen, die sich tagsüber zeigen und die durch eine Blasenfunktionsstörung oder rezidivierende Harnwegsinfekte erklärt werden könnten. Außerdem sind belastende und ungewöhnliche Ereignisse im Leben des Kindes ein wichtiger Aspekt, wie etwa ein neues Geschwisterchen oder der Verlust einer geliebten Person. Bei der körperlichen Untersuchung sollte unter anderem das Abdomen palpiert und auf angeborene Anomalien geachtet werden. Ist die nächtliche Urininkontinenz das einzige Symptom, ist lediglich eine Urinuntersuchung einschließlich einer mikrobiologischen Kultur erforderlich.

Die Behandlungsansätze reichen von Lebensstiländerungen über eine medika-

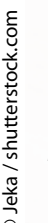

Jede Nacht eine neue Herausforderung: Siegt der Harndrang oder der Schlaf?

mentöse Behandlung bis hin zu alternativen Therapieansätzen. Da bei vielen Kindern das Selbstwertgefühl leidet, ist es besonders wichtig, dem Kind Verständnis und Zuversicht zu signalisieren. Die Eltern müssen wissen, dass ihr Kind den nächtlichen Harndrang einfach nicht unter Kontrolle hat, auch wenn es tagsüber keine Problem gibt. Sie müssen Wege suchen, mit dem Problem zurechtzukommen und ihrem Kind dennoch ein normales Leben zu ermöglichen.

Eine Hilfe sind Plastikunterlagen. Auch die abendliche Trinkmenge sollte begrenzt werden. Als wirksam hat sich die Therapie mit Desmopressin erwiesen. Der Wirkstoff erhöht die Wasserrückresorption und verringert damit die nächtliche Urinproduktion. Sobald er aber abgesetzt wird, ist das Problem wieder präsent. Auch trizyklische Antidepressiva wurden bei kindlichen Bettnässern eingesetzt. Sie zeigen zwar eine gewisse Wirkung, aber auch unerwünschte Nebenwirkungen.

Den größten Langzeitnutzen haben offenbar Bettnässer-Alarmsysteme wie eine Klingelmatte, die ein Warnsignal auslöst, sobald das Kind uriniert. Es soll durch den Alarm aufwachen und zur Toilette gehen. Über längere Zeit soll das Kind so lernen, auf seinen Blasendruck zu achten. Ein systematisches Review ergab, dass $66 \%$ der Kinder nach 10-20 Wochen 14 aufeinander folgende Nächte trocken blieben, verglichen mit $4 \%$ der Kinder ohne eine solche Hilfe. Von den vielen Versuchen mit alternativen Therapieverfahren wie Hypnotherapie, Akupunktur, chiropraktischer Behandlung oder Psychotherapie wurden bislang keine durchschlagenden Erfolge berichtet. Letztlich kann man auch darauf vertrauen, dass das Kind älter wird, denn bei $15 \%$ der Kinder pro Jahr verliert sich das nächtliche Problem auch ohne jede Behandlung.

Fazit: Mit der Unterstützung der Eltern und der richtigen Behandlung können Kinder lernen, ihren Blasendruck zu kontrollieren. Am nachhaltigsten wirken BettnässerAlarmsysteme. Dr. Christine Starostzik

Kiddoo DA. Noctural enuresis.

CMAJ 2012; 184: 908-11 